\title{
ITERATIVE SOURCE-CODED EQUALIZATION: TURBO ERROR CONCEALMENT FOR ISI CHANNELS
}

\author{
Laurent Schmalen, Thorsten Clevorn, Peter Vary \\ Institute of Communication Systems and Data Processing (ind) \\ RWTH Aachen University, Germany \\ E-Mail: $\{$ schmalen, vary $\}$ dind.rwth-aachen. de
}

\begin{abstract}
In this paper, we analyze the behavior of soft decision source decoding for ISI channels and present an iterative scheme similar to Turbo equalization allowing to combat efficiently intersymbol interference effects. In a first step, we investigate the behavior of different index assignments in the quantizer and show that gains are possible by iterative processing without any channel (de)coding. In a second step, we use short block codes resulting in redundant index assignments at the transmitter. The receiver does not perform any explicit channel decoding but exploits the additional redundancy of the index assignment in the soft decision source decoder. Furthermore, we show that a very simple channel precoder permits even larger gains without any additional decoding complexity. Moreover, the extension towards a flexible multi-mode system allowing a trade-off between channel quality and quantization quality over a wide range of channel conditions is presented.
\end{abstract}

\section{INTRODUCTION}

With the discovery of Turbo codes, channel coding close to the Shannon limit became possible with moderate computational complexity. In the past years, the Turbo principle of exchanging extrinsic information between separate channel decoders has also been adapted to other receiver components.

To exploit the residual redundancy in source-coded parameters such as scale factors or predictor coefficients for speech, audio, and video signals in a Turbo process, iterative source-channel decoding (ISCD) has been presented in [1], [2]. This residual redundancy occurs due to imperfect source encoding resulting for instance from the delay constraints.

Whenever the transmission of modulation symbols over a communication channel is distorted by intersymbol interference, equalization becomes indispensable. There exist several types of equalizers; in this paper however, we will only consider maximum a posteriori (MAP) equalization which reinterprets the ISI channel as a rate-1 convolutional code and uses a variation of the BCJR-algorithm [3] for equalization. The Turbo principle of exchanging extrinsic reliabilities has been adapted to equalization in [4] by interpreting the equalizer as the inner decoding component of a serially concatenated system. The resulting algorithm is called Turbo equalization. The use of channel precoders [5], [6] has enabled additional performance gains in Turbo equalization systems.

In this paper we propose a system similar to the ISCM system [7]. Instead of a higher order modulation, the inner component

Thorsten Clevorn is now with Infineon Technologies, COM Development Center NRW, Duisburg, Germany of the system comprises a channel exhibiting intersymbol interference (ISI) and the corresponding equalizer. The iterative loop at the receiver consists of equalization and soft decision source decoding (SDSD) [8] exploiting the redundancy remaining in the sourcecoded parameters. As the use of channel coding would result in an additional decoding module at the receiver and thus in a threefold iterative system, we propose a solution employing a special kind of channel coding by using short block codes as in [9]. Channel decoding is performed implicitly in the SDSD. Furthermore, the use of channel precoders permits additional gains with no increase in the decoder complexity.

In Section 2 we will give an overview of the system and present the different modules used, i.e., soft decision source decoding, implicit channel decoding with short block codes resulting in redundant index assignments, equalization, and channel precoding. Simulation results for a variety of setups are given in Section 3. Furthermore, the convergence behavior is analyzed using the EXIT chart technique [10], [11]. Simulation results of a multi-mode system similar to the ones in [9], [12] are given too.

\section{SYSTEM MODEL}

In the following, we will give an overview and a description of the iterative source-coded equalization (ISCE) system. In Section 2.1, we will introduce the entire system and we will briefly review the components of the system in Sections 2.2 and 2.3.

\subsection{Iterative Source-Coded Equalization (ISCE)}

In Fig. 1, the baseband model of the ISCE system is depicted. At time instant $\tau$, a source encoder determines a frame $\underline{u}_{\tau}$ of $K_{S}$ source codec parameters $u_{\kappa, \tau}$ with $\kappa \in\left\{1, \ldots, K_{S}\right\}$ denoting the position in the frame. The single elements $u_{\kappa, \tau}$ of $\underline{u}_{\tau}$ are assumed to be statistically independent from each other. Each value $u_{\kappa, \tau}$ is individually mapped to a quantizer reproduction level $\bar{u}_{\kappa, \tau}^{\left(\xi_{\kappa}\right)}$ with index $\xi_{\kappa} \in\left\{0, \ldots, Q_{\kappa}-1\right\}$. The number of quantizer levels is usually assumed as $Q_{\kappa}=2^{M_{\kappa}}$. To each quantizer level $\bar{u}_{\kappa, \tau}^{\left(\xi_{\kappa}\right)}$ selected at time instant $\tau$, a unique bit pattern $\mathbf{v}_{\kappa, \tau}$ of $M_{\kappa}$ bits is assigned according to the index assignment $\mathbf{v}_{\kappa, \tau}=\Gamma\left(\xi_{\kappa}\right)$. For simplicity, we assume $M_{\kappa}=M$ for all $\kappa$ and omit the time index $\tau$ in the following. The single bits of a bit pattern $\mathbf{v}_{\kappa}$ are indicated by $v_{\kappa}^{(m)}$, $m \in\{1, \ldots, M\}$. The frame of bit patterns is denoted by $\underline{\mathbf{v}}$. The interleaver $\pi$ scrambles the incoming frame $\underline{\mathbf{v}}$ of data bits in a deterministic manner. 


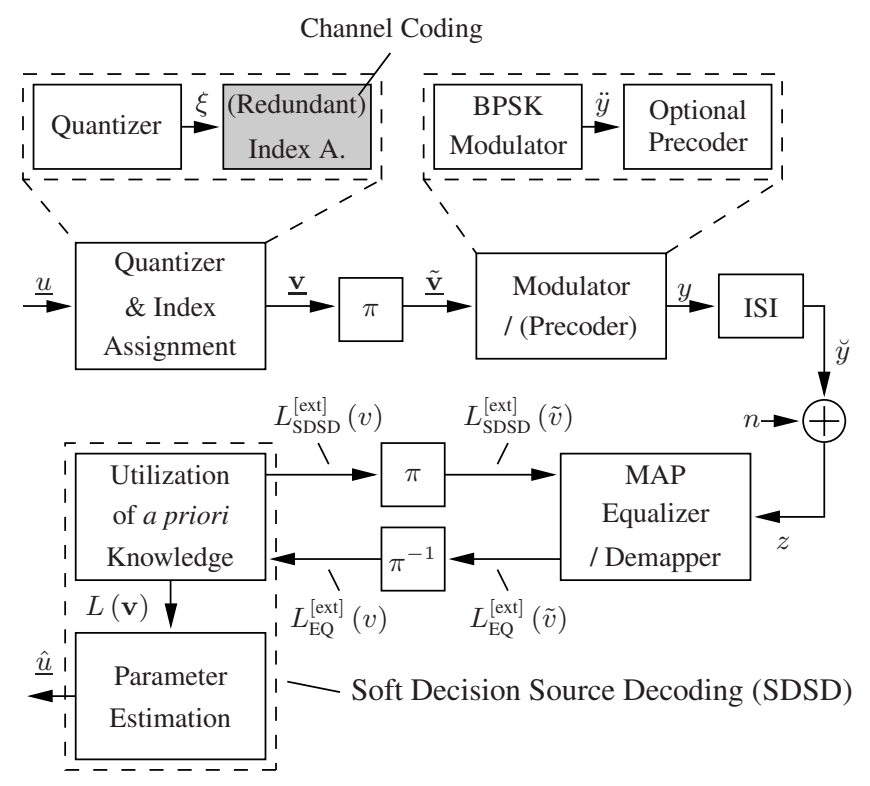

Fig. 1. Baseband model of the proposed ISCE scheme.

The interleaved frame is denoted $\underline{\tilde{\mathbf{v}}}$ with $M \cdot K_{S}$ bits $\tilde{v}_{k}$ indexed by $k, k \in\left\{1, \ldots, M \cdot K_{S}\right\}$, which are mapped to bipolar bits $\ddot{y}_{k} \in\{ \pm 1\}$ for BPSK transmission with symbol energy $E_{\mathrm{s}}$. Prior to transmission over the channel, the modulation symbols can optionally by precoded [5], [6]: $y_{k}=\ddot{y}_{k} \cdot y_{k-2}$ for $k \in\left\{3, \ldots, M \cdot K_{S}\right\}$ and $y_{k}=\ddot{y}_{k}$ for $k \in\{1,2\}$ (cmp. Fig. 2). In the case no precoding is performed, $y_{k}=\ddot{y}_{k}, k \in\left\{1, \ldots, M \cdot K_{S}\right\}$ holds. We only consider BPSK modulation in this paper in order to demonstrate the concept which can easily be extended to other modulation schemes such as QPSK or DPSK.

On the channel, the modulation symbols $y_{k}$ exhibit intersymbol interference due to the channel impulse response $h(\ell)=\sum_{i=0}^{C-1} h_{i} \delta(\ell-i)$ of finite length $C$. Additionally, complex additive white Gaussian noise (AWGN) $n_{k}=n_{k}^{\prime}+j n_{k}^{\prime \prime}$ with known power spectral density $\sigma_{n}^{2}=N_{0}\left(\sigma_{n^{\prime}}^{2}=\sigma_{n^{\prime \prime}}^{2}=N_{0} / 2\right)$ is applied, i.e.,

$$
z_{k}=n_{k}+\breve{y}_{k}=n_{k}+\sum_{i=0}^{C-1} h_{i} y_{k-i} .
$$

The received symbols $z_{k}$ are evaluated in a Turbo process which exchanges extrinsic reliabilities between equalizer (EQ) and soft decision source decoder (SDSD). Such reliability information can either be evaluated in terms of probabilities $P(\cdot)$ or in log-likelihood ratios, or, in short, $L$-values, $L(\cdot)$.

The equalizer utilized in the system depicted in Fig. 1 performs a maximum a posteriori (MAP) estimation and is based on the LogMAP algorithm [13]. For the equations for the computation of the extrinsic probabilities or their respective $L$-values in each component, we refer the reader to the literature, i.e., for the equalizer to [4], [11], [14], and for the SDSD (including parameter estimation with the minimum mean squared error serving as fidelity criterion) to [2], [8], [15].

In this paper we do not consider explicit channel coding and decoding as this would result in a multiple Turbo loop in the receiver yielding a more complex analysis of the system. Instead, robustness against transmission errors is ensured by adding artificial redundancy using a small block code resulting in redundant index assignments [9].

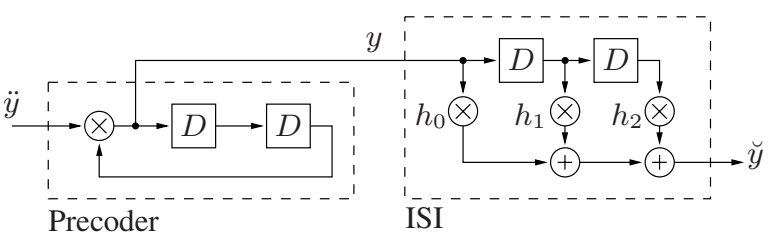

Fig. 2. Precoder $\left(G_{\text {pre }}(D)=1 /\left(1+D^{2}\right)\right)$ and $C=3$ tap ISI channel

\subsection{Redundant Index Assignments}

Redundant index assignments for ISCD were first proposed in [12]. A related concept for the non-iterative case has been presented in [16]. In comparison to a transmission scheme with explicit (outer) channel coding of rate $r_{\mathrm{C}}$, the number of bits assigned to each quantizer level $\bar{u}_{\kappa, \tau}^{\left(\xi_{\kappa}\right)}$ can be increased by a factor of $1 / r_{\mathrm{C}}$ if we assume a constant gross bit rate. So instead of using a typical $r_{C}=1 / 2$ channel encoder and, e.g., $M=3$ bits for the index assignment, bit patterns $\mathbf{v}$ of size $M^{\star}=M / r_{\mathrm{C}}=6$ can be assigned to each quantizer level. One possible choice would now be to use $Q^{\star}=2^{M^{\star}}=64$ quantizer levels to reduce the quantization noise. However, the only constraint for $Q^{\star}$ is $Q^{\star} \leq 2^{M^{\star}}$. Thus, if for instance the quantization noise for $Q^{\star}=8$ is specified as target, we can apply a redundant index assignment with $M^{\star}=6$ bits for each level. Such an index assignment can be considered as a (potentially non-linear) block code with the binary representation of the quantizer level index as input [9].

\subsection{Equalization and Precoding}

To combat the distortions caused by intersymbol interference (ISI) on transmission channels, equalization is absolutely necessary. Several types of equalizers exist, such as the zero forcing (ZF) or the MMSE equalizers [17]. In this paper, however, we will consider MAP equalization. The ISI channel, characterized by its impulse response $h(\ell)$ of length $C$, is modelled by an FIR filter with binary input $y \in\{ \pm 1\}$. Therefore, a binary channel trellis with $2^{C-1}$ states can be constructed and the well known Viterbi algorithm [17] or the BCJR algorithm [3] with modified metric increments can be used for equalization. For instance, the logarithmic metric increment $\log \gamma\left(s, s^{\prime}\right)$ used in a Log-MAP implementation [13] of the BCJRtype equalizer is given by [11]

$$
\log \gamma\left(s, s^{\prime}\right)=\frac{-1}{2 \sigma_{n}^{2}} \cdot\left(z_{k}-\sum_{i=0}^{C-1} h_{i} \breve{y}_{k-i}\right)^{2}+\frac{\ddot{y}_{k}}{2} \cdot L_{\mathrm{EQ}}^{[\mathrm{apri}]}\left(\ddot{y}_{k}\right)
$$

with $\breve{y}_{k-i}$ the hypothesized transmitted symbols according to the trellis state $s$, and $\ddot{y}$ the input symbol given by the considered state transition in the channel trellis. $L_{\mathrm{EQ}}^{[\mathrm{apri}]}(\ddot{y})$ is the a priori knowledge on bit level and in the ISCE scheme presented in Fig. $1, L_{\mathrm{EQ}}^{[\mathrm{apri}]}(\ddot{y})$ corresponds to $L_{\mathrm{SDSD}}^{[\mathrm{ext}}(\ddot{y})=L_{\mathrm{SDSD}}^{[\mathrm{ext}]}(\tilde{v})$.

Recent findings [18] have shown that the inner component of a serially concatenated Turbo scheme should be recursive and of rate 1. Thus, a recursive channel precoder [5], [6] with transfer function $G_{\text {pre }}(D)=1 /\left(1+D^{2}\right)$ as depicted in Fig. 2 may be used to enable the equalizer, i.e., the inner component of the receiver, to exploit a recursive transfer function and to improve the iterative decoding capabilities. In the case of bipolar bit representations, i.e., $\ddot{y} \in\{ \pm 1\}$ $(0 \mapsto+1 ; 1 \mapsto-1)$, the output of the channel precoder is given by

$$
y_{k}=\ddot{y}_{k} \cdot y_{k-2} \text {. }
$$




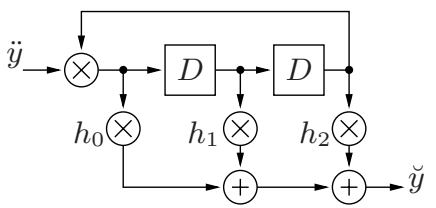

Fig. 3. Model of a $C=3$ tap ISI channel with precoding

Figure 3 depicts the recursive channel model for $C=3$ used to build the equalizer trellis in the case precoding according to (3) is used.

\section{SIMULATION RESULTS}

The capabilities of the proposed iterative source-coded equalization (ISCE) scheme are demonstrated by a simulation example. The parameter signal-to-noise ratio (SNR) between the originally generated parameters $u_{\kappa}$ and the reconstructed estimated parameters $\hat{u}_{\kappa}$ is used for quality evaluation. Instead of using any specific speech, audio, or video encoder, we model $K_{S}=250$ statistically independent source codec parameters $u_{\kappa}$ by $K_{S}$ independent $1^{\text {st }}$ order Gauss-Markov processes with auto-correlation $\rho=0.95$. These auto-correlation values can be observed in typical speech and audio codecs, e.g., for the scale factors in CELP codecs or MP3.

Each parameter $u_{\kappa}$ is scalarly quantized by a $Q$ or $Q^{\star}$ level Lloyd-Max quantizer using $M$ or $M^{\star}$ bits/parameter. As index assignment $\Gamma: \xi \mapsto \mathbf{v}$ serves either the natural binary index assignment (NB), the EXIT optimized index assignments $\left(\mathrm{EO}_{M}^{Q}\right)$ presented in [15], or the redundant block code based $\left(\mathrm{BC}_{M^{*}}^{Q^{\star}}\right)$ or non-linear block code based $\left(\mathrm{BCNL}_{M^{\star}}^{Q^{\star}}\right)$ index assignments presented in [9]. All used index assignments are summarized in Table 1. Note that the EXIT optimized bit mappings need to be optimized separately for each value of $\rho$.

Example: With $Q^{\star}=4, M^{\star}=6$, and $\Gamma$ : $\mathrm{BC}_{6}^{4}$ follows: $\xi=0 \mapsto\{\mathbf{v}\}_{10}=0 \quad \ldots \quad \xi=3 \longmapsto\{\mathbf{v}\}_{10}=54$ corresponding to the bit pattern $\mathbf{v}=(110110)$.

Table 1. Index assignments $\Gamma$ used for the simulations

\begin{tabular}{|l|l|}
\hline$\Gamma,(\cdot)_{M}^{Q}$ & $\{\mathbf{v}\}_{10}=\Gamma(\xi), \xi \in\{0, \ldots, Q-1\}$ \\
\hline $\mathrm{NB}_{3}^{8}$ & $0,1,2,3,4,5,6,7$ \\
$\mathrm{EO}_{3}^{8}$ & $6,3,5,0,7,2,4,1$ \\
\hline$\Gamma,(\cdot)_{M^{\star}}^{Q^{\star}}$ & $\{\mathbf{v}\}_{10}=\Gamma(\xi), \xi \in\left\{0, \ldots, Q^{\star}-1\right\}$ \\
\hline $\mathrm{BC}_{6}^{3}$ & $0,27,45$ \\
$\mathrm{BC}_{6}^{4}$ & $0,27,45,54$ \\
$\mathrm{BCNL}_{6}^{5}$ & $0,11,29,46,51$ \\
$\mathrm{BCNL}_{6}^{6}$ & $0,11,22,29,46,51$ \\
$\mathrm{BC}_{6}^{7}$ & $0,11,22,29,37,46,51$ \\
$\mathrm{BC}_{6}^{8}$ & $0,11,22,29,37,46,51,56$ \\
$\mathrm{BCNL}_{6}^{12}$ & $0,11,22,29,37,46,51,56,63,52,41,7$ \\
$\mathrm{BC}_{6}^{16}$ & $0,11,22,29,37,46,51,56,63,52,41,34,26,17,12,7$ \\
$\mathrm{BC}_{6}^{24}$ & $0,3,5,6,9,10,12,15,17,18,20,23,24,27,29,30,33$, \\
& $34,36,39,40,43,45,46$ \\
$\mathrm{BC}_{6}^{32}$ & $0,3,5,6,9,10,12,15,17,18,20,23,24,27,29,30,33$, \\
& $34,36,39,40,43,45,46,48,51,53,54,57,58,60,63$ \\
\hline
\end{tabular}

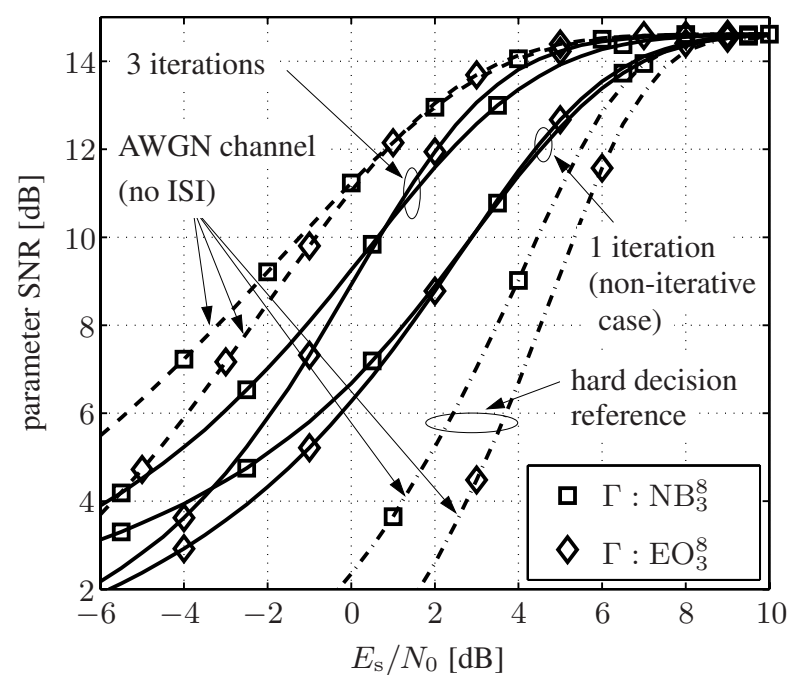

Fig. 4. Parameter SNR for ISCE with $M=3$ (no channel precoding)

The ISI channel used throughout this paper is a channel with $C=3$ coefficients $h_{0}=0.407, h_{1}=0.815$ and $h_{2}=0.407$ taken from [17].

\subsection{ISCE without Artificial Redundancy}

Simulation results for the basic ISCE system without precoding and without redundant index assignments are depicted in Fig. 4. As a reference, the dot and dash curves show the simulation results of a system using conventional hard decision source decoding (consisting of a simple table lookup) after transmission over a non-ISI AWGN channel. The dashed curves show the results of soft decision source decoding [8] after transmission over the non-ISI AWGN channel. They mark the upper bound of the reachable performance of the iterative system. In the non-iterative case, i.e., without exploring extrinsic feedback, the performance of the AWGN transmission cannot be reached if ISI is present. After 3 iterations however, a performance gain of approximately $2 \mathrm{~dB}$ can be observed. The system deploying the natural binary (NB) index assignment cannot utilize more than 2 iterations whereas the EXIT optimized (EO) index assignment can utilize up to 3 iterations.

If a parameter SNR design constraint of, e.g., $14 \mathrm{~dB}$ is given, the loss in terms of $E_{\mathrm{s}} / N_{0}$ compared to the undistorted transmission is only $0.8 \mathrm{~dB}$ (compared to $3.3 \mathrm{~dB}$ for the non-iterative case.
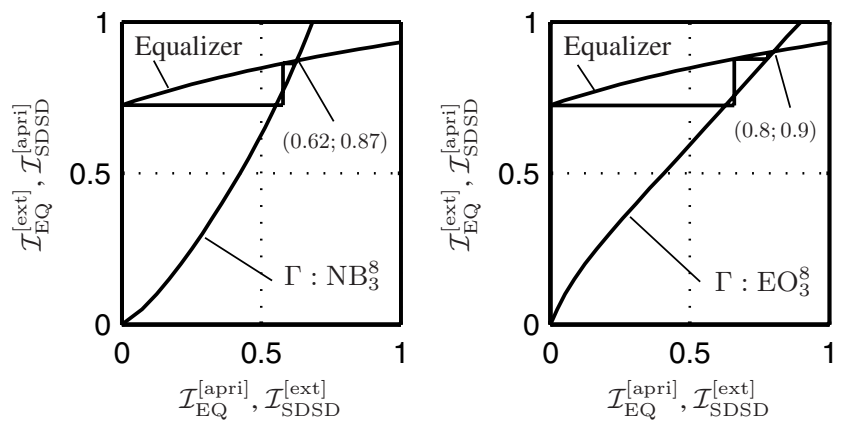

Fig. 5. EXIT charts for ISCE without artificial redundancy at $E_{\mathrm{s}} / N_{0}=3.5 \mathrm{~dB}$ (cmp. Fig. 4) 


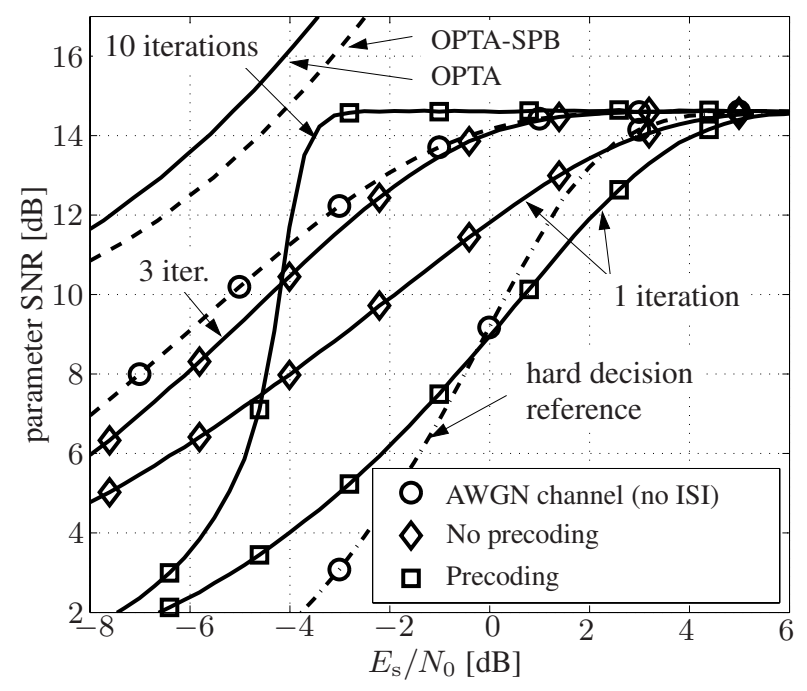

Fig. 6. Parameter SNR for ISCE with $M^{\star}=6$ and $Q^{\star}=8$ and index assignment $\mathrm{BC}_{6}^{8}$

In Fig. 5, the convergence behavior of ISCE at $E_{\mathrm{S}} / N_{0}=3.5 \mathrm{~dB}$ is analyzed using EXIT charts [10], [11]. It can clearly be seen that the use of the EXIT optimized index assignment enables the iterative decoder to reach a point closer to the upper right corner in the EXIT chart. Due to the characteristic of the SDSD, it is not possible to reach the rightmost point of the equalizer characteristic, the bound given by the non-ISI transmission [11]. Therefore, index assignments resulting in more favorable EXIT characteristics, i.e., an intersecting point towards the right, should be used.

\subsection{ISCE with Artificial Redundancy}

Simulation results for the ISCE system with implicit channel decoding via the redundant index assignment $\mathrm{BC}_{6}^{8}$ are depicted in Fig. 6. This system resembles the BC-TDeC system presented in [9], with the exception that the (de)modulator is replaced by the ISI channel, the optional precoder, and the equalizer. The dot and dash curve marks the reference consisting of a conventional hard decision source decoder and MAP decoding [13] of the block code after transmission over a non-ISI AWGN channel. The dashed curve gives the performance for the non-ISI channel if soft decision source decoding is used. In this case, channel decoding is done implicitly in the SDSD. If no channel precoding is used, the ISCE system can exploit up to three iterations and the performance of the transmission over a non-ISI channel can almost be reached for high parameter SNRs. This convergence behavior is visualized in the left EXIT chart of Fig. 7. After three iterations the intersection point has been reached. The EXIT characteristic of the SDSD with $\mathrm{BC}_{6}^{8}$ index assignment is more beneficial for the ISCE system as the intersection point with the equalizer characteristic is moved to the right.

If channel precoding is used, the non-iterative system performs worse than the non-precoded system. But precoding permits the decoder to exploit more than 3 iterations and after 10 iterations, a gain of over $3 \mathrm{~dB}$ at a parameter SNR of $14 \mathrm{~dB}$ compared to the noniterative, non-ISI-distorted transmission can be observed. Figure 6 also contains the Optimum Performance Theoretically Attainable (OPTA) [19], [20], adapted for the utilized 3-tap ISI channel and the limit considering the finite framesize of 1500 bit (OPTA-SPB) [19]. At a parameter SNR of $14 \mathrm{~dB}$ the loss in terms of $E_{\mathrm{S}} / N_{0}$ of the iterative system with precoding compared to the OPTA-SPB limit is only $\approx 1 \mathrm{~dB}$.
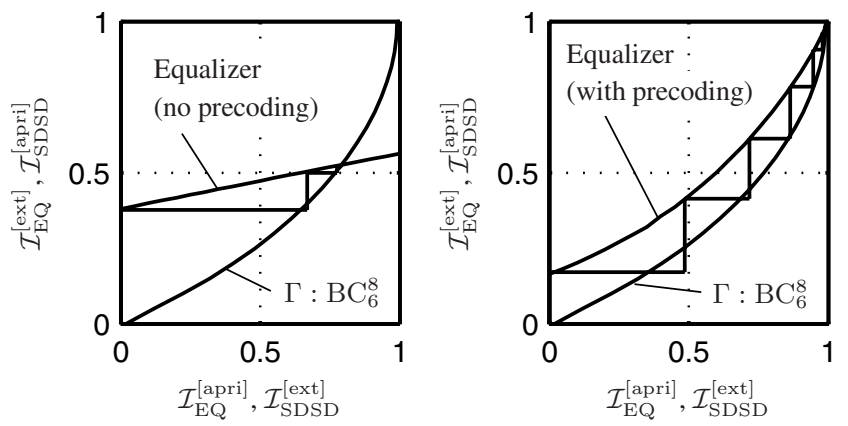

Fig. 7. EXIT charts for ISCE with redundant index assignment $\mathrm{BC}_{6}^{8}$ at $E_{\mathrm{s}} / N_{0}=-2 \mathrm{~dB}$ (cmp. Fig. 6)

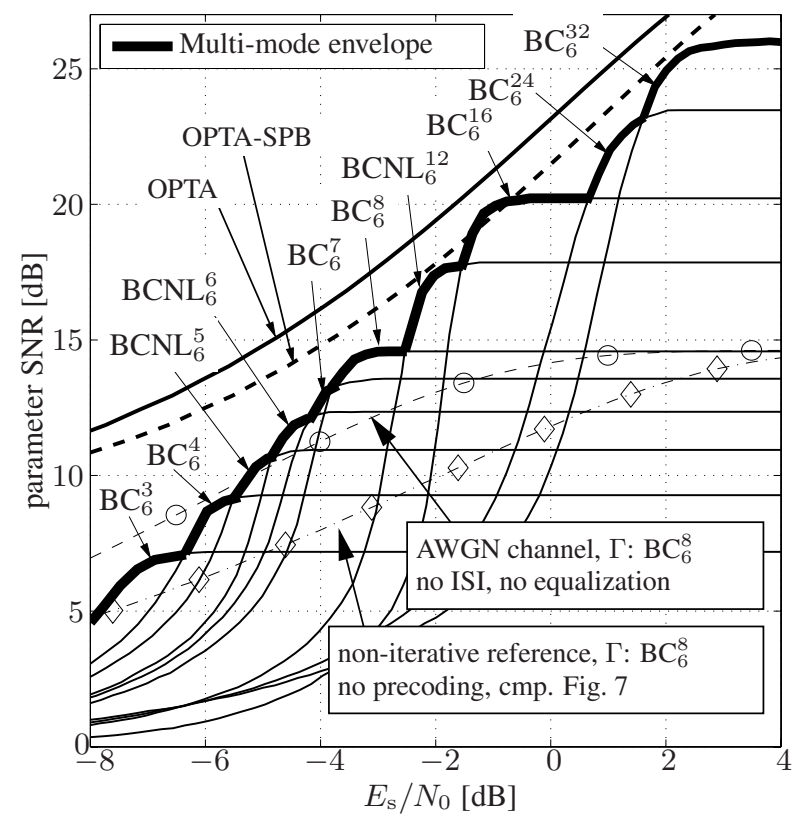

Fig. 8. Performance of multi-mode ISCE with $M^{\star}=6$ for 10 iterations

The convergence behavior is depicted in the right EXIT chart of Fig. 7. The equalizer considering the precoded channel shows quite a different slope than the non-precoded equalizer. Precoding allows the equalizer to reach the upper right corner of the EXIT chart and therefore enables perfect decoding if a sufficient number of iterations are carried out. Furthermore it can be observed that the slightly arcuated shapes of both characteristics match very well, featuring a narrow decoding tunnel and thus allowing the decoder to benefit from a large number of iterations.

Simulation results for the extension of the ISCE scheme to a multi-mode system as in [9], [12] are depicted in Fig. 8. This multi-mode system is based on the fact that several realizations of $Q^{\star} \leq 2^{M^{\star}}$ are possible for a given $M^{\star}$. The lower the number of $Q^{\star}$ is, the higher the error robustness can be. Of course, at the same time higher quantization noise has to be accepted in good channel conditions. Thus, adaptive mode-switching according to the channel condition is beneficial. Note that in contrast to multi-mode joint source-channel coding standards like the GSM-AMR (adaptive multi-rate), the second component, i.e., the ISI channel and the precoder, is not adapted in case of a dynamic dimensioning of $Q^{\star}$. The number $M^{\star} \cdot K_{S}$ of bits $v$ is the same for all realizations of $Q^{\star}$. 
When for every $E_{\mathrm{s}} / N_{0}$ always the best choice of the considered index assignments is selected, we obtain a multi-mode envelope (thick solid line). This multi-mode scheme gives superior performance in all cases. It features large quality gains in parameter SNR for good channels as well as robust transmission for bad channel conditions.

It can be seen in Fig. 8 that the multi-mode scheme performs superior over the whole depicted $E_{\mathrm{s}} / N_{0}$ range than the non-iterative reference system, i.e., if no feedback of extrinsic information is exploited. Compared with the non-ISI-distorted case, i.e., the transmission over a pure AWGN channel, parameter SNR gains can be observed for $E_{\mathrm{s}} / N_{0}>-5.3 \mathrm{~dB}$. The OPTA-SPB limit can be closely reached over a wide range of channel conditions and is even exceeded for $\mathrm{BC}_{6}^{16}$. The reason for this behavior is the utilization of past received data in the SDSD [19]. Obviously the multi-mode system shows two advantages: first, the envelope does not show a steep waterfall behavior but the degradation increases softly with decreasing $E_{\mathrm{s}} / N_{0}$; second, the effect of the quantization noise can be reduced for good channel conditions allowing better quantization of the parameters.

\section{CONCLUSION}

In this paper we proposed a Turbo equalization system for transmission of quantized source-coded parameters over a channel exhibiting intersymbol interference (ISI). If no channel coding is used, the iterative decoder consisting of equalizer and soft decision source decoder allows to reach quite closely the performance of the non-ISI transmission over an AWGN channel with soft decision source decoding.

If an ISCE system with short block codes is used, the added redundancy is not used for channel decoding but for soft decision source decoding. The channel encoding is realized by a redundant index assignment of the quantized parameters. Iterative decoding also allows in this case to reach quite closely the performance of the non-ISI distorted transmission. If a recursive channel precoder is used, we have shown that the iterative ISCE system allows gains of several $\mathrm{dB}$ compared to the non-iterative, non-distorted case. This behavior has been explained and confirmed by an EXIT chart analysis.

Finally, we presented a multi-mode system realized by varying the redundant index assignment. In this case, an optimal trade-off between quality and error robustness for different channel conditions can be realized.

\section{ACKNOWLEDGMENT}

Parts of this work have been financed by the European Union in the scope of the FP6 research project FlexCode.

\section{REFERENCES}

[1] M. Adrat, P. Vary, and J. Spittka, "Iterative Source-Channel Decoder Using Extrinsic Information from Softbit-Source Decoding," in IEEE ICASSP, Salt Lake City, USA, May 2001.

[2] N. Görtz, "On the Iterative Approximation of Optimal Joint Source-Channel Decoding," IEEE J. Select. Areas Commun., vol. 19, no. 9, pp. 1662-1670, Sept. 2001.

[3] L. R. Bahl, J. Cocke, F. Jelinek, and J. Raviv, "Optimal Decoding of Linear Codes for Minimizing Symol Error Rate," IEEE Trans. Inform. Theory, vol. 20, pp. 284-287, Mar. 1974.
[4] C. Douillard, M. Jézéquel, C. Berrou, A. Picart, P. Didier, and A. Glavieux, "Iterative Correction of Intersymbol Interference: Turbo-Equalization," European Trans. Telecomm., vol. 6, pp. 507-511, 1995.

[5] K. Narayanan, "Effect of Precoding on the Convergence of Turbo Equalization for Partial Response Channels," IEEE J. Select. Areas Commun., vol. 19, no. 4, pp. 686-698, Apr. 2001.

[6] I. Lee, "The Effect of a Precoder on Serially Concatenated Coding Systems with an ISI Channel," IEEE Trans. Commun., vol. 49, no. 7, pp. 1168-1175, July 2001.

[7] T. Clevorn, P. Vary, and M. Adrat, "Iterative Source Coded Modulation: Turbo Error Concealment by Iterative Demodulation,” in IEEE ICASSP, Montreal, Canada, May 2004.

[8] T. Fingscheidt and P. Vary, "Softbit Speech Decoding: A New Approach to Error Concealment," IEEE Trans. Speech Audio Processing, vol. 9, no. 3, pp. 240-251, Mar. 2001.

[9] T. Clevorn, M. Adrat, and P. Vary, "Turbo DeCodulation using Highly Redundant Index Assignments and Multi-Dimensional Mappings," in Intl. Symposium on Turbo Codes \& Related Topics, Munich, Germany, Apr. 2006.

[10] S. ten Brink, "Convergence Behaviour of Iteratively Decoded Parallel Concatenated Codes," IEEE Trans. Commun., vol. 49, no. 10, pp. 1727-1737, Oct. 2001.

[11] S. ten Brink, "Designing Iterative Decoding Schemes with the Extrinsic Information Transfer Chart," AEÜ Int. J. Electron. Commun., pp. 389-398, Dec. 2000.

[12] M. Adrat, P. Vary, and T. Clevorn, "Optimized Bit Rate Allocation for Iterative Source-Channel Decoding and its Extension towards Multi-Mode Transmission," in 14th IST Mob. \& Wirel. Comm. Summit, Dresden, Germany, June 2005.

[13] J. Hagenauer, E. Offer, and L. Papke, "Iterative Decoding of Binary Block and Convolutional Codes," IEEE Trans. Inform. Theory, vol. 42, no. 2, pp. 429-445, Mar. 1996.

[14] M. Tüchler, R. Koetter, and A. C. Singer, "Turbo Equalization: Principles and New Results," IEEE Trans. Commun., vol. 50, no. 5, pp. 754-767, May 2002.

[15] M. Adrat and P. Vary, "Iterative Source-Channel Decoding: Improved System Design Using EXIT Charts," EURASIP Jour. Appl. Sig. Proc., vol. 205, no. 6, pp. 928-941, May 2005.

[16] S. Heinen and P. Vary, "Source-Optimized Channel Coding for Digital Transmission Channels," IEEE Trans. Commun., vol. 53, no. 4, pp. 592-600, Apr. 2005.

[17] J. G. Proakis, Digital Communications, chapter 10, McGrawHill, New York, 4th edition, 2001.

[18] A. Ashikhmin, G. Kramer, and S. ten Brink, "Extrinsic Information Transfer Functions: Model and Erasure Channel Properties," IEEE Trans. Inform. Theory, pp. 2657-2673, Nov. 2004.

[19] Thorsten Clevorn, Laurent Schmalen, Peter Vary, and Marc Adrat, "On The Optimum Performance Theoretically Attainable for Scalarly Quantized Correlated Sources," in Proc. of ISITA, Seoul, Korea, Nov. 2006.

[20] D. Arnold and H.-A. Loeliger, "On the Information Rate of Binary-Input Channels with Memory," in Proc. of ICC, Helsinki, Finland, June 2001. 